\title{
Temperature-dependent efficiency droop of blue InGaN micro-light emitting diodes
}

\author{
Pengfei Tian, ${ }^{1}$ Jonathan J. D. McKendry, ${ }^{1}$ Johannes Herrnsdorf, ${ }^{1}$ Scott Watson, ${ }^{2}$ \\ Ricardo Ferreira, ${ }^{1}$ Ian M. Watson, ${ }^{1}$ Erdan Gu, ${ }^{1, a)}$ Anthony E. Kelly, ${ }^{2}$ and Martin D. Dawson ${ }^{1}$ \\ ${ }^{1}$ Institute of Photonics, University of Strathclyde, 106 Rottenrow, Glasgow G4 ONW, United Kingdom \\ ${ }^{2}$ School of Engineering, University of Glasgow, James Watt South Building, Glasgow G12 8LT, \\ United Kingdom
}

(Received 30 June 2014; accepted 21 October 2014; published online 29 October 2014)

\begin{abstract}
Temperature-dependent trends in radiative and Auger recombination coefficients have been determined at different injection carrier concentrations using InGaN micro-light emitting diodes $40 \mu \mathrm{m}$ in diameter. The differential lifetime was obtained first from the measured modulation bandwidth and was then employed to calculate the carrier concentration in the quantum well active region. When the temperature increases, the carrier concentration increases, but both the radiative and Auger recombination coefficients decrease. In addition, the temperature dependence of radiative and Auger recombination coefficients is weaker at a higher injection carrier concentration, which is strongly related to phase space filling. (C) 2014 AIP Publishing LLC.

[http://dx.doi.org/10.1063/1.4900865]
\end{abstract}

Significant progress has been achieved in the application and physical understanding of GaN-based light emitting diodes (LEDs). However, their external quantum efficiency (EQE) shows a non-thermal reduction at high injection current density, known as efficiency droop, which appears to be a barrier for the wide application of LEDs in general lighting. ${ }^{1,2}$ This phenomenon is generally studied under pulsed current conditions, which allows analysis of the effects of current density, independent of the heating. However, in many LED applications, such as automotive headlights, the ambient temperature is much higher than normal room temperature, and therefore, the study of temperature-dependent LED efficiency droop is necessary.

Recently, the issue of temperature-dependent reductions in $\mathrm{EQE}$, known as thermal droop, has received more attention. ${ }^{3}$ For example, a major reduction in EQE by about $30 \%$ has been observed from $300 \mathrm{~K}$ to $450 \mathrm{~K}^{3}$ Several groups have reported that at a fixed high current density of hundreds of $\mathrm{A} / \mathrm{cm}^{2}$, EQEs first increase and then decrease with increasing temperature, the detailed variation depending largely on the LED design. ${ }^{4-7}$ Different mechanisms have been proposed to explain the temperature-dependent efficiency droop, which is interdependent with currentdependent efficiency droop. The proposed current-dependent efficiency droop mechanisms include Auger recombination, electron leakage, and carrier delocalization. ${ }^{1,2}$ Recent significant experimental contributions include claims of direct observations of Auger electron emission under current injection $^{8,9}$ or photoluminescence. ${ }^{10}$ Within the framework of an $A B C$ recombination model, the temperature dependence of carrier recombination coefficients has often been investigated experimentally by fitting the temperature-dependent internal quantum efficiency (IQE) curves without considering electron leakage. ${ }^{11,12}$ With the increase in temperature, the defect-related Shockley-Read-Hall (SRH) recombination coefficient $A$ increases and the radiative recombination

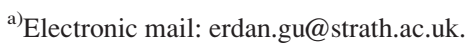

coefficient $B$ decreases. However, inconsistent temperaturedependent trends in the third-order coefficient $C$ (assumed hereon for concreteness to correspond to Auger recombination) have been reported, including a monotonous increase with increasing temperature ${ }^{11}$ and little variation at temperatures higher than $200 \mathrm{~K}^{12}$ In these studies, currentindependent $B$ and $C$ coefficients have been assumed, and their variations with injection current density/carrier concentration due to phase space filling effects have not been considered. Similar controversy also exists in the theoretical work based on direct/indirect Auger recombination calculations. ${ }^{13-16}$ Theoretical work has indicated that the temperature dependence of $B$ and $C$ coefficients varies at different carrier concentration in quantum wells (QWs) with different carrier confinement conditions; ${ }^{13}$ however, experimental verification of such predictions has not yet been achieved.

In this study, we experimentally determine temperaturedependent trends in carrier recombination coefficients, in particular the Auger coefficient, as a function of carrier concentration, through measurements of the experimental modulation bandwidth and EQE of blue-emitting InGaN micro-LEDs. Due to their excellent current spreading and heat dissipation ability, micro-LEDs can be operated at current densities up to several $\mathrm{kA} / \mathrm{cm}^{2}$ with little self-heating, as emphasized in previous publications. ${ }^{17,18}$ At such high injection current densities, the phase space filling effect is significant and its effect on the temperature-dependent $B$ and $C$ coefficients has been demonstrated here. In addition, the excellent current spreading of micro-LEDs helps to alleviate the effect of current crowding on carrier recombination at different current densities and temperatures. Furthermore, we use temperature-dependent modulation bandwidth for carrier recombination analysis. These bandwidth measurements follow methodologies developed in recent studies on the emerging application of visible light communications (VLC). ${ }^{19,20}$ While VLC using GaN-based LEDs operating at different temperatures has been demonstrated, ${ }^{21}$ the temperature-dependent mechanisms affecting performance 
in this application require further investigation to complement understanding of efficiency droop effects, commonly considered in a direct current (DC) drive context.

Individually addressable top-emission (through the $p$-contact) micro-LEDs on $c$-plane sapphire substrates with a diameter of $40 \mu \mathrm{m}$ and emission wavelength $\sim 445 \mathrm{~nm}$ were fabricated from a commercial wafer. The epistructure consists of an $n$-GaN layer, an $\mathrm{InGaN} / \mathrm{GaN}$ multiple quantum well (MQW) active region, an $\mathrm{AlGaN}$ current blocking layer, and a $p$-GaN layer. The micro-LED fabrication processes are similar to those in Ref. 22.

A previous study on micro-LEDs of comparable design shows that the junction temperature of a $40 \mu \mathrm{m}$ micro-LED differs insignificantly from the ambient temperature even under high injection DC, due to highly effective current spreading and heat dissipation. ${ }^{17,18}$ To confirm this observation, the light output power of the micro-LED was compared under pulsed current with low duty cycle (pulse width: $25 \mu$ s; pulse period: $525 \mu \mathrm{s}$ ) and DC. No difference of light output power was found up to a current of $35 \mathrm{~mA}\left(\sim 2800 \mathrm{~A} / \mathrm{cm}^{2}\right)$. Also, for a $40 \mu \mathrm{m}$ micro-LED from Ref. 17, the junction temperature shows little change until current density $\sim 2800 \mathrm{~A} / \mathrm{cm}^{2}$. Thus, the self heating can be neglected here at DC $35 \mathrm{~mA}$ or less for the measurements of light output power and bandwidth in this work. ${ }^{17,18} \mathrm{In}$ addition, potential effects of the temperature on current spreading as reported by Kudryk et al. ${ }^{23}$ are neglected due to the more uniform current spreading in micro-LEDs compared to broad-area LEDs.

The ambient temperature of the micro-LEDs was adjusted by placing the devices on a hotplate, and the temperature was monitored by bonding a thermocouple to the device. The optical $-3 \mathrm{~dB}$ modulation bandwidth was measured by combining a DC bias from a power source and a small-signal modulation of fixed amplitude from an HP8753ES network analyzer using a bias-tee. Then the light emission from the micro-LED was collected using a $1.4 \mathrm{GHz}$ bandwidth photoreceiver, and the frequency response was recorded by a network analyzer. To calculate the EQE, the light output power as a function of current under different temperatures was measured by a Si detector at a fixed distance from the sample. Detector response changes due to micro-LED spectral changes at different currents/temperatures were further considered to correct the measured light output power. Then, the light output power measured in an integrating sphere at room temperature $(\sim 300 \mathrm{~K})$ was used to calibrate the fraction of the light captured by the detector during the variable-temperature measurements.

Fig. 1(a) shows the temperature-dependent EQE versus current density characteristics on a semi-logarithmic scale with device temperature varied from 300 to $500 \mathrm{~K}$ in $25 \mathrm{~K}$ increments. It can be seen that with the increase in temperature, the EQE peak shifts towards higher current densities and the peak EQE decreases. At low current densities, less than $10 \mathrm{~A} / \mathrm{cm}^{2}$, the EQEs drop with increasing temperature. At high current densities, the variation is complicated. For example, at $1000 \mathrm{~A} / \mathrm{cm}^{2}$, from a close inspection of the data, we found that the EQE increases from 300 to $375 \mathrm{~K}$ and decreases from 375 to $500 \mathrm{~K}$. The electrical-to-optical bandwidths of the micro-LED are shown in Fig. 1(b). The bandwidths from 300 to $425 \mathrm{~K}$ demonstrate a clear trend. For
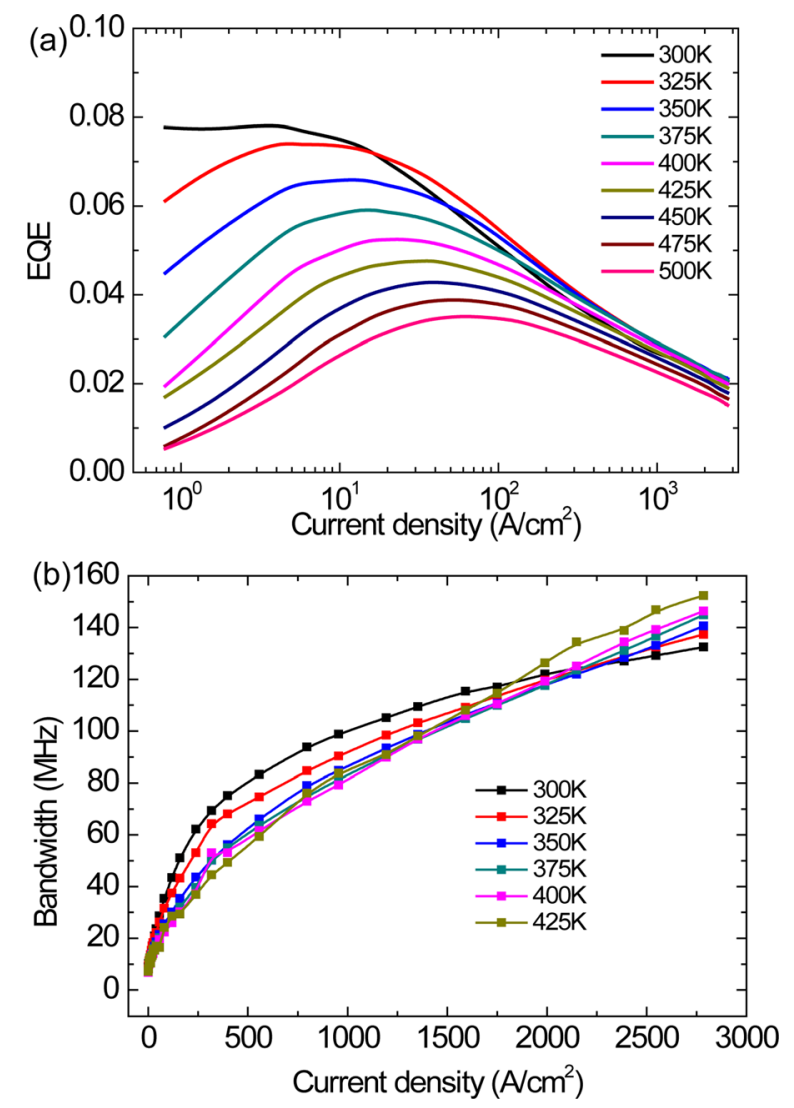

FIG. 1. (a) EQE versus current density from 300 to $500 \mathrm{~K}$ with $25 \mathrm{~K}$ temperature increments on a semi-logarithmic scale. (b) Bandwidth versus current from 300 to $425 \mathrm{~K}$ to show the trend with temperature. The bandwidths from $450 \mathrm{~K}$ to $500 \mathrm{~K}$ show large fluctuations and are not shown here.

example, at $500 \mathrm{~A} / \mathrm{cm}^{2}$, the bandwidth decreases, and, at $2500 \mathrm{~A} / \mathrm{cm}^{2}$, the bandwidth increases with the increase in temperature. The bandwidths from $450 \mathrm{~K}$ to $500 \mathrm{~K}$ show large fluctuations and are not shown here. It should be noted that three randomly selected micro-LEDs have been measured and the results confirm that the obtained temperaturedependent trends in $\mathrm{EQE}$ and bandwidth are experimentally repeatable.

The carrier concentration $n$ can be determined from the bandwidth measurement by equation ${ }^{19,24}$

$$
n=\frac{1}{q V_{\text {active }}} \int_{0}^{I} \eta_{\text {inj }}\left(I^{\prime}\right) \tau\left(I^{\prime}\right) d I^{\prime}
$$

where $q$ is the electron charge, $V_{\text {active }}$ is the effective volume of QWs in the active region, $I$ is the current, $\eta_{i n j}$ is the carrier injection efficiency affected by electron leakage, and $\tau$ is the differential lifetime calculated from the $-3 \mathrm{~dB}$ bandwidth, i.e., $f_{3 d B}=\sqrt{3} / 2 \pi \tau$. $V_{\text {active }}$ may change under different currents/temperatures due to carrier distribution in more QWs, indium fluctuation, and more possible factors. ${ }^{25-27}$ However, due to the lack of sufficient recombination volume information from available data, ${ }^{25-27}$ an accurate correction for different currents and temperatures is very challenging. Our analysis follows the common assumption, ${ }^{28,29}$ supported by many simulation studies, ${ }^{30,31}$ that only the QW closest to the $p$-GaN is heavily populated with carriers. Therefore, in this 


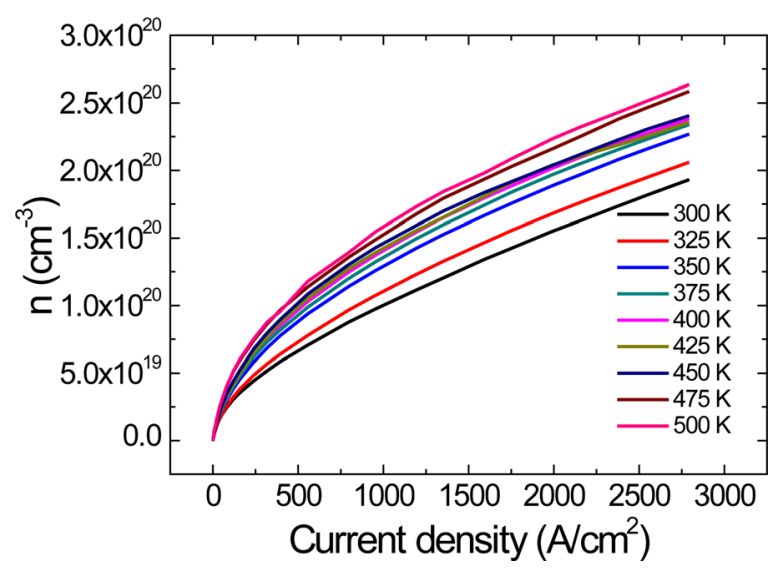

FIG. 2. Carrier concentration n versus current density from 300 to $500 \mathrm{~K}$.

work, one QW with a thickness of $2.8 \mathrm{~nm}$ is used to estimate $V_{\text {active }}$. In addition, carrier injection efficiency $\eta_{i n j}$ is assumed to be 1 for all temperatures. ${ }^{11,12}$ The electron leakage has little effect at low current densities (roughly before the EQE peak), but may affect LED efficiency at high current densities (roughly after the EQE peak). ${ }^{1}$ The latter determined temperature-dependent trend in carrier recombination coefficients is consistent in the whole current density range, so we consider that the effect of electron leakage on our results can be neglected. The calculated carrier concentrations $n$ at different temperatures are shown in Fig. 2. Generally, $n$ keeps increasing from 300 to $500 \mathrm{~K}$, which is consistent with the reduced recombination coefficients in the following text.

In the framework of the $A B C$-approximation, the EQE of the micro-LED is expressed by the following equation: ${ }^{1}$

$$
E Q E=E X E \times I Q E=E X E \times \frac{B n^{2}}{A n+B n^{2}+C n^{3}},
$$

where EXE is the LED light extraction efficiency. Following Refs. 19 and 32, a constant EXE value of $\sim 18 \%$ has been assumed here, for all current densities and temperatures in this work. ${ }^{11,12}$ In addition, a slightly different EXE value will not affect the trends in the $A B C$ coefficients. In Eq. (2), the $B$ and $C$ coefficients are only constant at low current densities owing to phase space filling effect. ${ }^{33,34}$ The $B$ and $C$ coefficients can be described by the empirical formulas $B=B_{0} /\left(1+n / N^{*}\right)$ and $C=C_{0} /\left(1+n / N^{*}\right)$, respectively. ${ }^{14,34}$ Here, $N^{*}$ is the phase space filling parameter, and $B_{0}$ and $C_{0}$ represent $B$ and $C$ at very low $n$, respectively. From the experimental EQE and IQE, the carrier recombination rates can be obtained. The total carrier recombination rate is expressed as $R_{\text {recom }}=I / q V_{\text {active }}$, which is the sum of radiative recombination rate $R_{r}=R_{\text {recom }} I Q E$ and nonradiative recombination rate $R_{n r}=R_{\text {recom }}(1-I Q E)$. Then, from $R_{n r} / n=A+C n^{2}$, the $A$ coefficient is obtained at low $n$ (e.g., $n=1 \times 10^{18} \mathrm{~cm}^{-3}$ ); from $R_{r} / n^{2}=B$, the $B$ coefficient is calculated; through $R_{n r} / n^{3}=A / n^{2}+C$, the $C$ coefficient is available at high $n$ (e.g., $n=2 \times 10^{20} \mathrm{~cm}^{-3}$ ). ${ }^{34,35}$ By fitting these curves as shown in Figs. 3(a) and 3(b), the parameters $A, B_{0}, C_{0}$, and $N^{*}$ can be obtained at different temperatures, as shown in Table I. The physical mechanisms underlying the trends in the fitted parameters are discussed as follows.
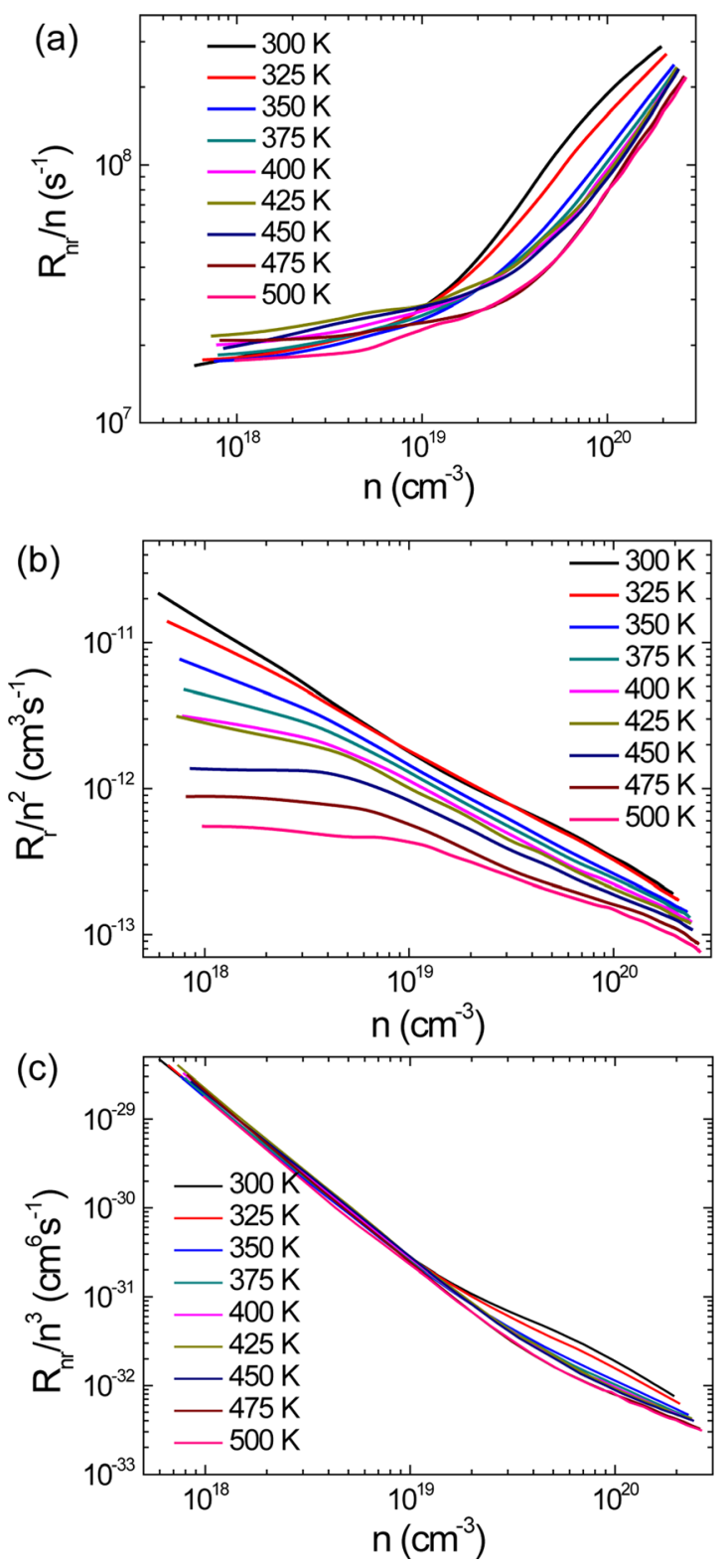

FIG. 3. The experimental variation of (a) $R_{n r} / n$, (b) $R_{r} / n^{2}$, (c) $R_{n r} / n^{3}$ with temperature from 300 to $500 \mathrm{~K}$.

At low current densities, the SRH recombination coefficient $A$ in Table $\mathrm{I}$, as determined from Fig. 3(a), shows a general increasing trend with increasing temperature, consistent with published results. ${ }^{28,36}$ However, scatter from the overall

TABLE I. Summary of fitted parameters $A, B_{0}, C_{0}$, and $N^{*}$ from 300 to $500 \mathrm{~K}$.

\begin{tabular}{lcccc}
\hline \hline$T(\mathrm{~K})$ & $A\left(\mathrm{~s}^{-1}\right)$ & $B_{0}\left(\mathrm{~cm}^{3} \mathrm{~s}^{-1}\right)$ & $C_{0}\left(\mathrm{~cm}^{6} \mathrm{~s}^{-1}\right)$ & $N^{*}\left(\mathrm{~cm}^{-3}\right)$ \\
\hline 300 & $1.74 \times 10^{7}$ & $8.76 \times 10^{-11}$ & $7.85 \times 10^{-30}$ & $1.94 \times 10^{17}$ \\
325 & $1.78 \times 10^{7}$ & $2.72 \times 10^{-11}$ & $1.90 \times 10^{-30}$ & $6.72 \times 10^{17}$ \\
350 & $1.76 \times 10^{7}$ & $1.15 \times 10^{-11}$ & $6.96 \times 10^{-31}$ & $1.42 \times 10^{18}$ \\
375 & $1.86 \times 10^{7}$ & $5.97 \times 10^{-12}$ & $3.19 \times 10^{-31}$ & $2.86 \times 10^{18}$ \\
400 & $2.03 \times 10^{7}$ & $3.65 \times 10^{-12}$ & $1.82 \times 10^{-31}$ & $4.75 \times 10^{18}$ \\
425 & $2.21 \times 10^{7}$ & $3.52 \times 10^{-12}$ & $1.97 \times 10^{-31}$ & $4.34 \times 10^{18}$ \\
450 & $2.03 \times 10^{7}$ & $1.57 \times 10^{-12}$ & $7.50 \times 10^{-32}$ & $1.17 \times 10^{19}$ \\
475 & $2.09 \times 10^{7}$ & $9.53 \times 10^{-13}$ & $5.05 \times 10^{-32}$ & $1.55 \times 10^{19}$ \\
500 & $1.77 \times 10^{7}$ & $5.51 \times 10^{-13}$ & $2.60 \times 10^{-32}$ & $3.18 \times 10^{19}$ \\
\hline \hline
\end{tabular}


trend exists, which may come from measurement errors in the $-3 \mathrm{~dB}$ bandwidths at very low currents. The possible reasons are the relatively large modulation signal compared to the DC component, as well as the noises of optical frequency response. Subsequent analysis has also shown that the temperature dependence of $A$ is relatively weak compared to those of $B$ and $C$.

In Fig. 3(b), the $B$ coefficient $\left(R_{r} / n^{2}\right)$ decreases with increasing temperature at all carrier concentrations. This is induced by phase space filling, i.e., the carrier distribution is approximately described by Maxwell-Boltzmann statistics at low carrier concentration and by Fermi-Dirac carrier distribution at high carrier concentration. ${ }^{14,23,33}$ As more carriers occupy states in higher sub-bands at higher temperatures, the carrier number per $\mathrm{d} \boldsymbol{k}$ interval in $\boldsymbol{k}$-space decreases with increasing temperature, leading to a reduced $B$ coefficient at higher temperatures. In addition, $N^{*}$ increases with increasing temperature as shown in Table I, i.e., phase-space filling occurs at a higher injection level at a higher temperature, which agrees with the theoretical and experimental trends in other publications ${ }^{14,23,33}$ but shows a stronger temperature dependence. The phase space filling effect in Ref. 14 and 33 was estimated by extracting the theoretical data of $B$ versus $n$. This can be understood by noting that at higher temperatures the thermal distribution already leads to a significant occupation of high- $\boldsymbol{k}$ states. Therefore, a significant change in the occupation of high- $\boldsymbol{k}$ states requires a higher carrier concentration than at lower temperatures.

Figs. 4(a) and 4(b) show the temperature variation of $B$ coefficient at $n=2 \times 10^{18} \mathrm{~cm}^{-3}$ and $n=1 \times 10^{20} \mathrm{~cm}^{-3}$, respectively. A strong decrease in $B$ with increasing temperature has been found at the low carrier concentration $n=2 \times 10^{18} \mathrm{~cm}^{-3}$, empirically scaling as $B \propto 1 / T^{4}$. However, at the high carrier concentration $n=1 \times 10^{20} \mathrm{~cm}^{-3}$, a weaker temperature dependence of $B \propto 1 / T^{3 / 2}$ appears. This observation agrees with the theoretical calculation very well ${ }^{13}$ and is
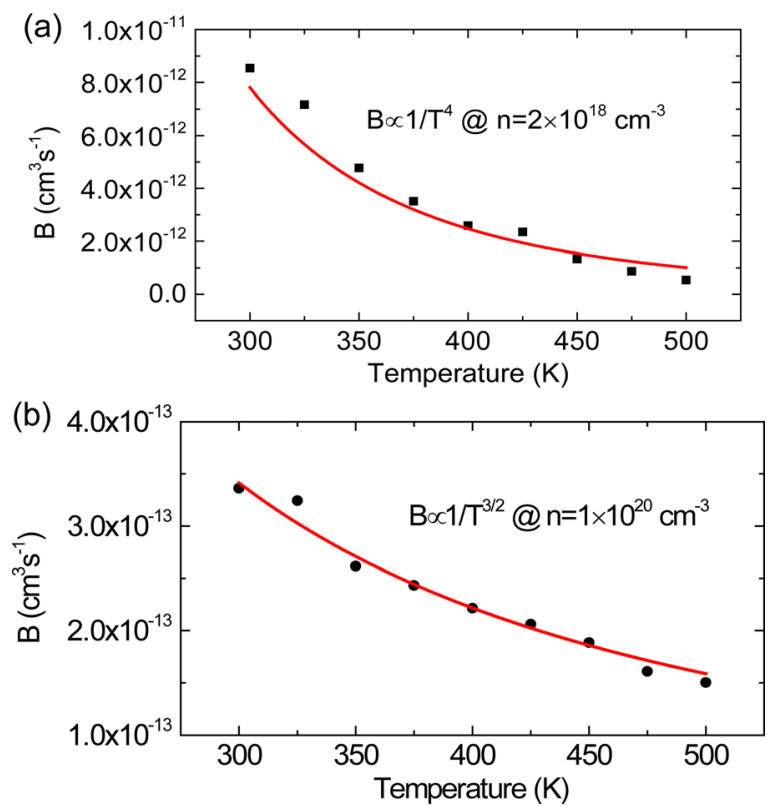

FIG. 4. B coefficient variation with temperature (a) at $n=2 \times 10^{18} \mathrm{~cm}^{-3}$ $\left(B \propto 1 / T^{4}\right)$ and (b) at $n=1 \times 10^{20} \mathrm{~cm}^{-3}\left(B \propto 1 / T^{3 / 2}\right)$. The red lines are fits to the experimental data (black symbols). explained as follows. At a low injection carrier concentration $\left(2 \times 10^{18} \mathrm{~cm}^{-3}\right)$, a fraction of carriers, close to the Fermi level and in the high energy tail of the occupation function, is strongly influenced by temperature; at a high carrier concentration $\left(1 \times 10^{20} \mathrm{~cm}^{-3}\right)$, the carrier states close to the bandgap are filled, so most carriers are at energies below the Fermi level, leading to less temperature dependence. ${ }^{13}$ Other experimental and theoretical work reported weak dependences of $B$ on $T,{ }^{11,14}$ which agrees with our results at high carrier concentrations.

The temperature-dependent $C$ coefficients as shown in Fig. 5(a) were calculated from the values of $C_{0}$ and $N^{*}$ in Table I. At all carrier concentrations, the $C$ coefficient decreases with the increase in temperature. In addition, similar to the case of radiative recombination, a weaker temperature dependence is obtained with increasing carrier concentration, which is again related to the phase space filling effect. The temperature dependence of $C$ at a carrier concentration of $1 \times 10^{20} \mathrm{~cm}^{-3}$ is shown in Fig. 5(b) and empirically follows $C \propto 1 / T^{2}$. The temperature dependence of the $C$ coefficient in this work is contrary to most theoretical and experimental trends in the literature, ${ }^{11,14-16}$ but agrees with one design in theoretical result with relatively weak carrier confinement in the QWs. ${ }^{13}$

In summary, the temperature dependence of the recombination coefficients in blue-emitting InGaN/GaN MQW LEDs has been investigated experimentally. With increasing temperature, the radiative and Auger recombination coefficients decrease. It is also found that the temperature dependence of radiative and Auger recombination coefficients becomes weaker at higher carrier concentrations. The phase space filling effect is proposed to contribute significantly to the variation of temperature-dependent radiative and Auger recombination coefficients. This work not only provides further insight into efficiency droop, but also helps to elucidate
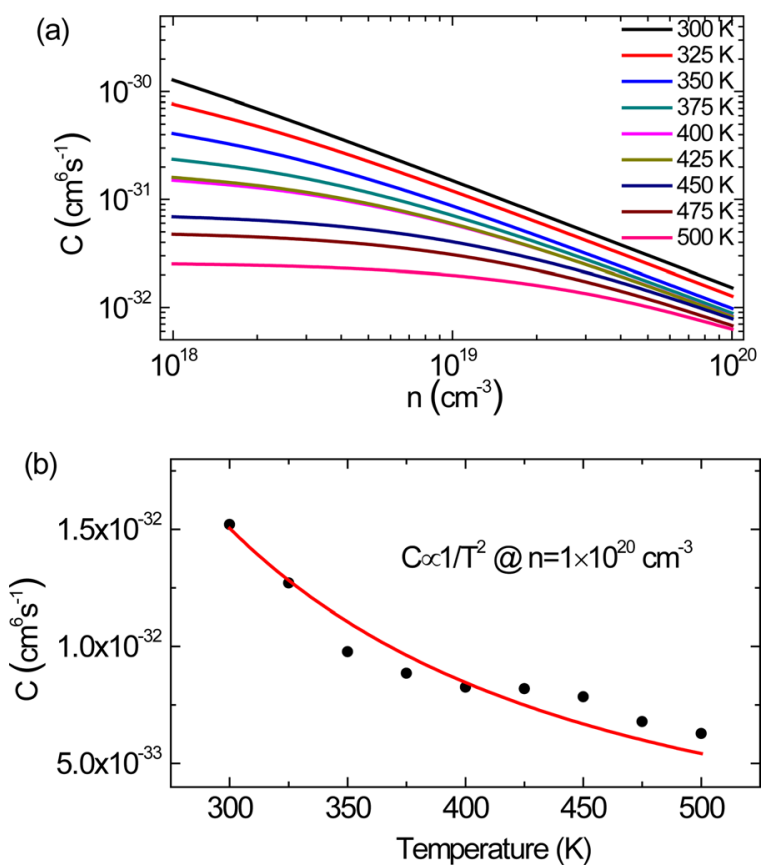

FIG. 5. (a) Calculated $\mathrm{C}$ coefficient versus $\mathrm{n}$ at different temperatures. (b) $\mathrm{C}$ coefficient variation with temperature at $n=1 \times 10^{20} \mathrm{~cm}^{-3}(C \propto 1 / T)$. The red line is a fit to the calculated data (black symbols). 
trends in the small-signal modulation bandwidth relevant to the application of LEDs in VLC.

We acknowledge support from Scottish Universities Physics Alliance, China Scholarship Council, Overseas Research Students Awards Scheme, University of Strathclyde, and Engineering and Physical Sciences Research Council Grant EP/K00042X/1. We thank Michael Wallace, Strathclyde University Department of Physics, for his help with the integrating sphere test.

${ }^{1}$ J. Piprek, Phys. Status Solidi A 207, 2217 (2010).

${ }^{2}$ G. Verzellesi, D. Saguatti, M. Meneghini, F. Bertazzi, M. Goano, G. Meneghesso, and E. Zanoni, J. Appl. Phys. 114, 071101 (2013).

${ }^{3}$ D. S. Meyaard, Q. Shan, J. Cho, E. F. Schubert, S.-H. Han, M.-H. Kim, C. Sone, S. J. Oh, and J. K. Kim, Appl. Phys. Lett. 100, 081106 (2012).

${ }^{4}$ H. Masui, T. Ive, M. C. Schmidt, N. N. Fellows, H. Sato, H. Asamizu, S. Nakamura, and S. P. DenBaars, Jpn. J. Appl. Phys., Part 1 47, 2112 (2008).

${ }^{5}$ D.-S. Shin, D.-P. Han, J.-Y. Oh, and J.-I. Shim, Appl. Phys. Lett. 100, 153506 (2012).

${ }^{6}$ D. S. Meyaard, G.-B. Lin, J. Cho, E. F. Schubert, H. Shim, S.-H. Han, M.H. Kim, C. Sone, and Y. S. Kim, Appl. Phys. Lett. 102, 251114 (2013).

${ }^{7}$ C. H. Wang, J. R. Chen, C. H. Chiu, H. C. Kuo, Y.-L. Li, T. C. Lu, and S. C. Wang, IEEE Photonics Technol. Lett. 22, 236 (2010).

${ }^{8}$ J. Iveland, L. Martinelli, J. Peretti, J. S. Speck, and C. Weisbuch, Phys. Rev. Lett. 110, 177406 (2013).

${ }^{9}$ J. Iveland, M. Piccardo, L. Martinelli, J. Peretti, J. W. Choi, N. Young, S. Nakamura, J. S. Speck, and C. Weisbuch, Appl. Phys. Lett. 105, 052103 (2014).

${ }^{10}$ M. Binder, A. Nirschl, R. Zeisel, T. Hager, H.-J. Lugauer, M. Sabathil, D. Bougeard, J. Wagner, and B. Galler, Appl. Phys. Lett. 103, 071108 (2013).

${ }^{11}$ B. Galler, P. Drechsel, R. Monnard, P. Rode, P. Stauss, S. Froehlich, W. Bergbauer, M. Binder, M. Sabathil, B. Hahn, and J. Wagner, Appl. Phys. Lett. 101, 131111 (2012).

${ }^{12}$ S. Y. Karpov, Phys. Status Solidi RRL 4, 320 (2010).

${ }^{13}$ J. Hader, J. V. Moloney, and S. W. Koch, IEEE J. Quantum Electron. 44,185 (2008).

${ }^{14}$ E. Kioupakis, Q. Yan, D. Steiauf, and C. G. Van de Walle, New J. Phys. 15, 125006 (2013).

${ }^{15}$ E. Kioupakis, P. Rinke, K. T. Delaney, and C. G. Van de Walle, Appl. Phys. Lett. 98, 161107 (2011).
${ }^{16}$ F. Bertazzi, M. Goano, and E. Bellotti, Appl. Phys. Lett. 101, 01111 (2012).

${ }^{17}$ Z. Gong, S. Jin, Y. Chen, J. McKendry, D. Massoubre, I. M. Watson, E. Gu, and M. D. Dawson, J. Appl. Phys. 107, 013103 (2010).

${ }^{18}$ P. Tian, J. J. D. McKendry, Z. Gong, B. Guilhabert, I. M. Watson, E. Gu, Z. Chen, G. Zhang, and M. D. Dawson, Appl. Phys. Lett. 101, 231110 (2012).

${ }^{19}$ R. P. Green, J. J. D. McKendry, D. Massoubre, E. Gu, M. D. Dawson, and A. E. Kelly, Appl. Phys. Lett. 102, 091103 (2013).

${ }^{20}$ J. J. D. McKendry, D. Massoubre, S. Zhang, B. R. Rae, R. P. Green, E. Gu, R. K. Henderson, A. E. Kelly, and M. D. Dawson, IEEE J. Lightwave Technol. 30, 61 (2012).

${ }^{21}$ J.-W. Shi, H.-W. Huang, F.-M. Kuo, J.-K. Sheu, W.-C. Lai, and M. L. Lee, IEEE Photonics Technol. Lett. 22, 1033 (2010).

${ }^{22}$ P. Tian, J. J. D. McKendry, Z. Gong, S. Zhang, S. Watson, D. Zhu, I. M. Watson, E. Gu, A. E. Kelly, C. J. Humphreys, and M. D. Dawson, J. Appl. Phys. 115, 033112 (2014).

${ }^{23}$ Y. Y. Kudryk, A. K. Tkachenko, and A. V. Zinovchuk, Semicond. Sci. Technol. 27, 055013 (2012).

${ }^{24}$ R. Olshansky, C. Su, J. Manning, and W. Powazinik, IEEE J. Quantum Electron. 20, 838 (1984).

${ }^{25}$ B. Galler, A. Laubsch, A. Wojcik, H. Lugauer, A. Gomez-Iglesias, M. Sabathil, and B. Hahn, Physica Status Solidi C 8, 2372 (2011).

${ }^{26}$ Y.-R. Wu, R. Shivaraman, K.-C. Wang, and J. S. Speck, Appl. Phys. Lett. 101, 083505 (2012).

${ }^{27}$ H.-Y. Ryu, D.-S. Shin, and J.-I. Shim, Appl. Phys. Lett. 100, 131109 (2012).

${ }^{28}$ D. S. Meyaard, Q. Shan, Q. Dai, J. Cho, E. F. Schubert, M.-H. Kim, and C. Sone, Appl. Phys. Lett. 99, 041112 (2011).

${ }^{29}$ Q. Dai, Q. Shan, J. Wang, S. Chhajed, J. Cho, E. F. Schubert, M. H. Crawford, D. D. Koleske, M.-H. Kim, and Y. Park, Appl. Phys. Lett. 97, 133507 (2010).

${ }^{30}$ J. R. Chen, Y. C. Wu, S. C. Ling, T. S. Ko, T. C. Lu, H. C. Kuo, Y. K. Kuo, and S. C. Wang, Appl. Phys. B 98, 779 (2010).

${ }^{31}$ Y.-K. Kuo, T.-H. Wang, J.-Y. Chang, and M.-C. Tsai, Appl. Phys. Lett. 99, 091107 (2011).

${ }^{32}$ T.-X. Lee, K. Gao, W.-T. Chien, and C.-C. Sun, Opt. Express 15, 6670 (2007).

${ }^{33}$ J. Hader, J. V. Moloney, and S. W. Koch, Appl. Phys. Lett. 87, 201112 (2005).

${ }^{34}$ A. David and M. J. Grundmann, Appl. Phys. Lett. 96, 103504 (2010).

${ }^{35}$ A. David and M. J. Grundmann, Appl. Phys. Lett. 97, 033501 (2010).

${ }^{36}$ H. P. T. Nguyen, M. Djavid, K. Cui, and Z. Mi, Nanotechnology 23, 194012 (2012). 